\title{
Competência Cultural: uma Resposta Necessária para Superar as Barreiras de Acesso à Saúde para Populações Minorizadas
}

\author{
Cultural Competence: an Answer Required to \\ Overcome Barriers to Health Care Access for \\ Minoritized Populations
}

Eneline A. H. Gouveia ${ }^{I \oplus}$

Rodrigo de Oliveira Silva

Bruno Henrique Soares Pessoa

\section{PALAVRAS-CHAVE}

- Medicina de Família.

- Competência Cultural.

- Currículo.
O reconhecimento das características culturais dos grupos sociais e de suas diferentes necessidades e concepções do processo saúde-doença, segundo Starfield, é um importante atributo derivado da atenção primária: a competência cultural. Por meio desse atributo, é possível desenvolver laços fortes com as pessoas e famílias alvo dos cuidados em saúde, obtendo-se maior satisfação, diagnósticos mais precisos e maior adesão ao tratamento. Mesmo num país como o Brasil, que ainda oferece um sistema de saúde universal e gratuito, populações minorizadas - por etnia, identidade de gênero, orientação sexual ou condição socioeconômica - enfrentam barreiras de acesso a esses serviços e apresentam piores indicadores de saúde. Tanto o profissional de saúde, quanto o usuário podem pertencer a vários grupos culturais simultaneamente, de acordo com sua identidade de gênero, idade, etnia, região, religião, orientação sexual, profissão ou papel que desempenha na família, classe social, bem como de outras características, e manifestar-se de maneiras culturalmente distintas em diferentes situações. É importante que o médico de família e comunidade reconheça que possui um conjunto de premissas e valores fundamentais que influenciam seu comportamento e sua interpretação do que é dito pelo outro. Devido a essa complexidade, esses aspectos devem ser considerados na discussão do processo saúde-doença e da construção de um plano de cuidados compartilhado. Este ensaio discute o desenvolvimento da competência cultural como uma resposta às barreiras de acesso à saúde para populações minorizadas no contexto da medicina de família e comunidade, com base na literatura. Foi realizada uma revisão exploratória do tema, que também identificou objetivos de aprendizagem e estratégias de ensinagem que podem ser incorporadas aos programas de residência em medicina de família e comunidade, visando colaborar com a sistematização do ensino desta competência, respeitando-se as especificidades regionais. Ele ainda aponta a necessidade de mudanças institucionais para incentivar e valorizar a prática da competência cultural, com disponibilização de tempo e infraestrutura para o desenvolvimento dessa competência pelos profissionais da atenção primária. 


\section{KEY-WORDS}

- Family Practice.

- Cultural Competence.

- Curriculum

Recebido em 10/3/19

Aceito em 10/6/19
ABSTRACT

The recognition of cultural characteristics of social groups, as well as their different needs and conceptions of the health-disease process is, according to Starfield, one of the important attributes derived from primary care: cultural competence. Through this attribute it is possible to develop strong bonds with the people and families who are the target of health care, resulting in greater satisfaction, more accurate diagnoses and greater adherence to treatment. Even in a country like Brazil, which still offers a free universal health system, minoritized populations - whether by ethnicity, gender identity, sexual orientation or socioeconomic status - face barriers to access these services and report poorer health indicators. Both the health professional and the user can belong to several cultural groups simultaneously, according to their gender, age, ethnicity, regionality, religion, sexual orientation, profession, role in the family, social class, as well as other characteristics and manifest in culturally distinct ways in different situations. It is important for the family physician to recognize the set of fundamental assumptions and values which influence their behavior and their interpretation of what is said by the other. Due to this complexity, these aspects should be considered in the discussion of the health-disease process and the construction of a shared care plan. This essay aims to discuss the development of cultural competence as a response to barriers health care access for minorities in the context of family and community medicine. An exploratory review of the literature was carried out, which also identified learning objectives and teaching strategies that can be incorporated into family and community medicine residency programs, aiming to collaborate with the systematization of teaching of this competence, respecting regional specificities. It also points out the need for institutional changes so that there is encouragement and appreciation of the practice of cultural competence, with the provision of time and infrastructure for the development of this competence by primary care professionals.

\section{COMPETÊNCIA CULTURAL IMPORTA?}

Desde que se tem registro, o Brasil ocupa uma posição de destaque quanto à desigualdade social. Aproximadamente $30 \%$ da renda do Brasil estão nas mãos de apenas $1 \%$ dos habitantes ${ }^{1}$. Essa desigualdade tem raízes históricas no processo de colonização e repercute na maioria dos problemas enfrentados pela população hoje, inclusive na falta de acesso a serviços de saúde ${ }^{2}$.

A declaração de Alma-Ata previa saúde para todos no ano 2000. No entanto, 40 anos se passaram e metade da população mundial ainda não tem acesso a nenhum tipo de serviço de saúde ${ }^{3}$. Mesmo num país como o Brasil, que ainda oferece um sistema de saúde universal e gratuito, populações minoritárias - seja por etnia, identidade de gênero, orientação sexual ou condição socioeconômica - ainda enfrentam barreiras de acesso a esses serviços e apresentam piores indicadores de saúde ${ }^{4}$. As pessoas negras, por exemplo, têm pior autoavaliação de saúde e estão mais expostas a viver em domicílios com condições precárias quando comparadas à população hegemônica branca $^{5}$. Além disso, sofrem mais com a falta de equilíbrio nas relações de poder na dinâmica médico-paciente, muitas vezes por preconceito e falta de treinamento desses profissionais para atuar com pessoas de diferentes cenários culturais ${ }^{6}$.

A população LGBT também enfrenta obstáculos, como atendimento discriminatório dos profissionais de saúde - seja em hospitais ou em unidade de saúde da família -, condutas inadequadas, constrangimentos, conotações preconceituosas ou mesmo ofensas verbais proferidas pelos profissionais ${ }^{7,8}$ As pessoas em situação de rua, embora apresentem maior prevalência de ISTs e doenças mentais, por vezes se recusam a procurar os serviços de saúde devido ao frequente mau atendimento e por terem, inúmeras vezes, acesso negado a estabelecimentos de saúde ${ }^{9}$. Entre os indígenas, a mortalidade infantil ainda é quase três vezes maior do que a média nacional, e mais da metade dos óbitos de bebês indígenas são provocados por doenças respiratórias, parasitárias e nutricionais ${ }^{10}$. Segundo o Ministério da Saúde do Brasil ${ }^{10}$ :

[...] ainda é possível observar limitações no planejamento e gestão das ações e serviços para o alcance da cobertura ideal 
de saúde aos povos indígenas, em especial, no que se refere à atenção básica às mulheres, crianças e adolescentes indígenas, quando comparados com a cobertura da população nacional. A maior parte dos problemas relacionados à garantia do acesso e da qualidade no atendimento em terras indígenas está relacionada às dificuldades legais, logísticas e operacionais para instalação de infraestrutura adequada, deslocamento das equipes, abastecimentos de insumos e medicamentos, além da falta de preparo dos profissionais para atuar em contextos interculturais.

O reconhecimento das características culturais dos grupos sociais e de suas diferentes necessidades e concepções do processo saúde-doença é, segundo Starfield, um importante atributo derivado da atenção primária: a competência cultural ${ }^{11}$. Por meio desse atributo é possível desenvolver laços fortes com as pessoas e famílias alvo dos cuidados em saúde, obtendo-se maior satisfação, diagnósticos mais precisos e maior adesão ao tratamento ${ }^{12}$. Apesar dos esforços do governo federal para diminuir a desigualdade em saúde mediante políticas públicas com o Programa Mais Saúde (2006) - como a Política Nacional de Saúde Integral da População Negra, Política Nacional de Saúde Integral das Populações do Campo e da Floresta, Política Nacional de Saúde Integral de Lésbicas, Gays, Bissexuais, Travestis e Transexuais, Política Nacional para a População em Situação de Rua ${ }^{13}$ - e antes mesmo, por meio da criação de um Subsistema de Saúde Indígena (1992) ${ }^{14}$, as normativas ainda são incapazes de garantir que a cultura seja utilizada como um critério para a consolidação de práticas que valorizem as diversas interpretações e modos de intervenção na área da saúde ${ }^{15}$. Nesse contexto, fica evidente a necessidade de treinamento adequado dos profissionais de saúde para o desenvolvimento da competência cultural.

Este ensaio tem por objetivo a discussão do desenvolvimento da competência cultural como uma resposta às barreiras de acesso à saúde para populações minorizadas. Assim, foi realizada uma revisão exploratória da literatura, utilizando-se os descritores "competência cultural" nas bases de dados PubMed e Google Scholar, e a seleção dos artigos foi feita pelos autores segundo a relevância para com o tema. $\mathrm{O}$ tema começa sendo explorado de uma perspectiva mais ampla, segundo o próprio conceito de cultura, perpassa estratégias para o desenvolvimento da competência cultural e resulta numa compilação de objetivos de aprendizagem identificados como relevantes, apresentados como produto final.

\section{MAS O QUE É CULTURA, AFINAL?}

Talvez devido a sua complexidade, o conceito de cultura é amiúde reduzido à ideia de etnicidade ou raça. No entanto, enquanto etnicidade faz referência a uma origem comum de um grupo, bem como à autodefinição por parte do indivíduo, a palavra raça está mais associada a rótulos aplicados por outros, com base nas características físicas observadas num grupo determinado de pessoas e se tornou ultrapassada quando faz referência a seres humanos, sendo mantida quando trata de "igualdade racial" ou "preconceito racial"16. Já as concepções de cultura são muito mais amplas e diversas, não havendo um consenso entre os antropologistas com relação a sua definição até o momento ${ }^{17}$.

A cultura possui limites imprecisos e está submetida a mudanças graduais ao longo do tempo devido a sua distribuição psicológica e social. Ela afeta o comportamento e a interpretação do comportamento humano, bem como processos biológicos. Se diferencia da natureza humana, que é universal - algo comum a todos os seres humanos - e da personalidade única de cada indivíduo, sendo uma construção individual e coletiva da sociedade ${ }^{18}$. Um dos conceitos de cultura que melhor se enquadra na discussão da competência cultural é o de Spencer-Oatey ${ }^{18}$.

\footnotetext{
Um conjunto difuso de premissas e valores fundamentais, orientações para a vida, crenças, políticas, procedimentos e convenções comportamentais que são compartilhadas por um grupo de pessoas e que influenciam (mas não determinam) o comportamento de cada membro e suas interpretações do "significado" do comportamento de outras pessoas.
}

O médico de família e comunidade (MFC) em seu papel de comunicador, tendo como base o Método Clínico Centrado na Pessoa (MCCP) - no exercício diário de entender a doença, a experiência da doença e a pessoa como um todo -, precisa também reconhecer que seu conjunto de premissas e valores fundamentais influencia seu comportamento e sua interpretação do que é dito pelo outro. Podemos compreender melhor o grau de complexidade dessa relação se entendermos ainda que tanto o MFC quanto a pessoa alvo do seu cuidado podem pertencer a vários grupos culturais simultaneamente, segundo sua identidade de gênero, idade, etnia, região, religião, orientação sexual, profissão ou papel que desempenha na família, classe social, bem como de outras características, e manifestar-se de maneiras culturalmente distintas em diferentes situações ${ }^{18}$. Assim, é importante considerar também cada um desses aspectos no momento de discutir sobre saúde, doença e de construir o plano de cuidados compartilhado ${ }^{19}$. 
Segundo Weaver ${ }^{20}$, a cultura pode ser analisada em diferentes camadas, como um iceberg. A parte mais superficial, a parte "visível" da cultura, seria o comportamento humano. Aquilo que se exterioriza e que é possível observar, no entanto, muitas vezes é difícil de interpretar, como expressões artísticas, modo de comer, vestir e se relacionar com os outros. O segundo nível, já submerso, seria a motivação para determinado comportamento e está relacionada a normas, objetivos e valores. Seria a interpretação que as próprias pessoas fazem de seu comportamento, e portanto consciente, podendo ser acessada por meio do diálogo. Mas, para de fato entender uma cultura, é necessário ir ainda mais profundo e alcançar as motivações ocultas nas quais os valores e normas se baseiam. Essas premissas podem ser acessadas se o MFC souber fazer as perguntas "certas" durante a entrevista clínica. São premissas fundamentais, relacionadas ao que as pessoas entendem sobre a natureza humana, relacionamentos humanos, educação de filhos, conceito de tempo e espaço, o que é bom ou ruim, certo ou errado, aceitável ou inaceitável. Crenças acerca do que é a natureza e a causa das doenças ou do bem-estar ${ }^{20}$.

\section{E VOCÊ TEM COMPETÊNCIA CULTURAL PARA CUIDAR DAS PESSOAS?}

Competência cultural pode ser definida como a "capacidade de realizar um cuidado efetivo, compreensivo e respeitoso, de maneira compatível com as crenças e práticas culturais de saúde do usuário, e no idioma de sua preferência". Esse termo, criado no cenário americano, é o mais utilizado na literatura, no contexto da saúde, para se referir à relação intercultural médico-pessoa. Além dessa definição,existem muitas outras de acordo com diferentes origens ${ }^{21}$. Campinha-Bacote ${ }^{22}$ define competência cultural como:

o processo pelo qual um profissional de saúde se empenha para se tornar capaz de trabalhar adequadamentelefetivamente dentro do contexto cultural da pessoa, família ou comunidade que necessita de seus cuidados. Sendo resultado, antes de tudo, necessário haver um desejo/uma motivação cultural para o desenvolvimento de consciência cultural, habilidades culturais e encontros culturais.

Essa dinâmica está ilustrada na Figura 1.

Segundo Murray-Garcia ${ }^{23}$, num contexto onde generalizações, estereótipos, privilégios e racismo estão arraigados na sociedade, e também presentes na relação médico-paciente, a competência cultural poderia ser mais bem descrita como "humildade cultural":

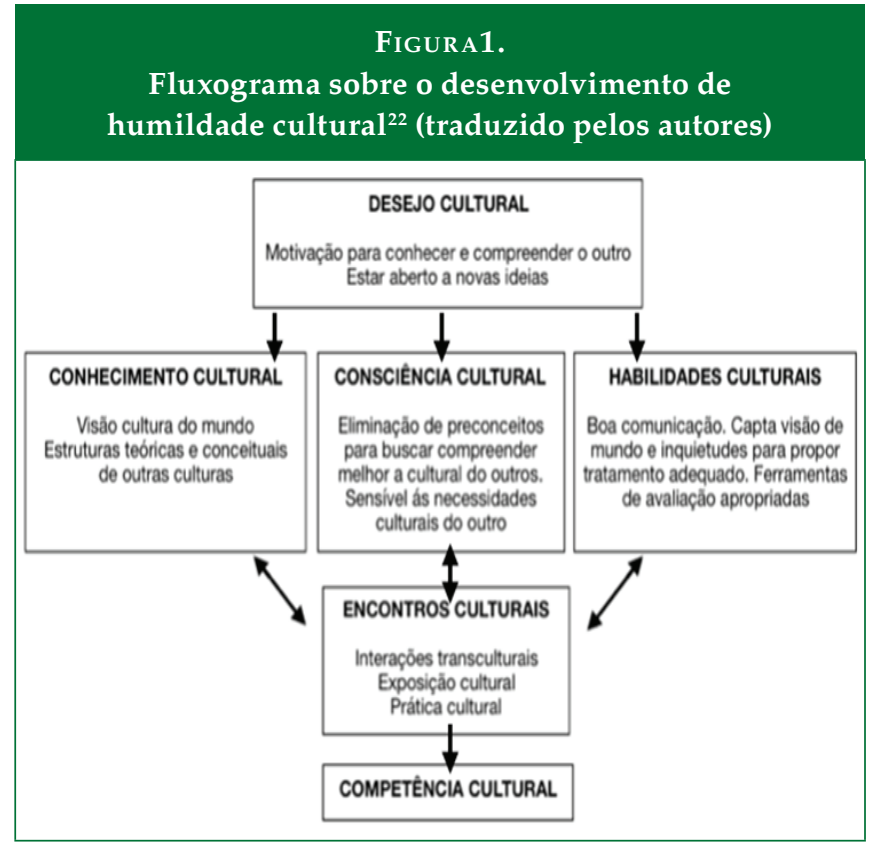

Um compromisso vitalício de autoavaliação e autocrítica, para os desequilíbrios de poder existentes na relação médico-paciente; desenvolver parcerias clínicas de benefícios mútuos; e advocacia não paternalista com as comunidades, em nome de indivíduos e populações.

Nessa concepção, a competência cultural possui seis componentes: mente aberta, consciência de si, "ausência de ego", autorreflexão, autocrítica e solidariedade ${ }^{24}$.

Nesse mesmo período, na Nova Zelândia, foi desenvolvido o conceito de "segurança cultural", a partir do contexto Maori. Esse conceito carrega uma responsabilidade política e social, relacionada à saúde negligenciada de um povo, demandando equidade na prestação de cuidados pelo sistema de saúde. Enfoca o empoderamento dos usuários e a diminuição da desigualdade existente na relação de poder médico-paciente. Diversas organizações e sistemas de saúde no mundo têm adotado esse conceito para a melhoria da assistência a populações minorizadas ${ }^{25}$.

Uma prática de saúde culturalmente segura é caracterizada por ações que reconhecem, respeitam e alimentam a identidade cultural única de um povo e atendem às suas necessidades, expectativas e direitos. Ela acontece quando um profissional de saúde desenvolve sensibilidade cultural e é capaz de identificar e refletir sobre sua própria cultura e a influência que ela exerce sobre sua prática. Dessa maneira, a interação dos dois não é prejudicial, e a dignidade e o respeito são mantidos para ambos ${ }^{26}$. Não se trata de uma lista de 
verificação ou memorização de normas diferentes baseadas no grupo cultural, mas de entender a pessoa à sua frente $\mathrm{e}$ estabelecer uma relação de confiança, considerando a interferência das opressões históricas e das relações de poder nesses relacionamentos ${ }^{27}$.

Diante do exposto, podemos afirmar que o treinamento do MFC em competência cultural, priorizando uma prática antirracista, crítica e autorreflexiva para identificação dos preconceitos inconscientes, é uma tarefa necessária e urgente. Embora não haja sistematização ou padronização do ensino dessa competência pelas instituições, que na maioria das vezes acontece de maneira empírica e informal ${ }^{28}$, as evidências apontam que esse treinamento aumenta a satisfação dos usuários, podendo melhorar a aderência ao tratamento ${ }^{29,30}$.

O processo de desenvolvimento da competência cultural pode ser descrito como um contínuo através do qual o profissional pode evoluir de forma positiva ou negativa ao longo dos anos, dependendo do seu empenho para a aquisição e manutenção dessa competência ${ }^{31}$. Essa autoavaliação pode ser feita por meio de uma escala representada pelo Quadro 1.

Na discussão do desenvolvimento da competência cultural, a habilidade de comunicação é um componente essencial. Ter o método clínico centrado na pessoa (MCCP) como uma das principais ferramentas do MFC permite evitar erros comuns numa relação terapêutica intercultural. Martinez sistematiza alguns erros aos quais devemos estar atentos ${ }^{32}$
(Quadro 2). No entanto, é importante lembrar que apenas o domínio do método clínico centrado na pessoa não é suficiente para alcançar a competência cultural. Isto porque, apesar das muitas interseções entre o MCCP e a competência cultural, e de ambos se destacarem nos últimos dez anos como elementos necessários à melhoria da qualidade dos sistemas de saúde, apresentam enfoques diferentes. Enquanto o MCCP prioriza o cuidado personalizado, com o olhar voltado ao indivíduo e visando à qualidade do cuidado para todos de maneira igualitária, a competência cultural visa à equidade no cuidado em saúde, tendo como principal foco as populações minorizadas ${ }^{33}$.

Assim, além da habilidade de se fazer entender - reconhecendo a necessidade de aprimorar comunicação, criatividade e flexibilidade -, a competência cultural é também composta por mais dois domínios: o domínio cognitivo, que engloba conhecimento da epidemiologia e manifestações de doenças em diversos grupos e conhecimento dos diferentes efeitos que os tratamentos podem desencadear em diferentes grupos étnicos, além do conhecimento sobre a história e cultura de cada grupo; e o domínio atitudinal, relacionado à consciência de como a cultura modela o comportamento e a maneira de pensar, e reconhecer que temos o nosso próprio preconceito e tendência a estereótipos ${ }^{34}$.

O processo de aprendizagem da competência cultural é mais complexo do que o desenvolvimento de sensibilidade cultural e autoconhecimento. Isto porque há certa resistência dos

QuAdro 1

Breve resumo do continuum da competência cultural. Adaptado de Terrell RD e Lindsey RB. Culturally Proficient Leadership: The Personal Journey Begins Within. 2009 (traduzido pelos autores)

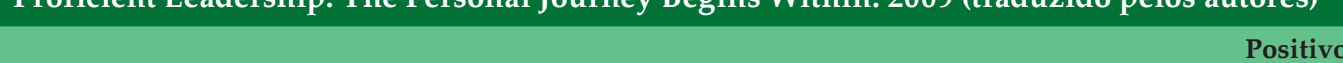

\begin{tabular}{|c|c|c|c|}
\hline & & & $\begin{array}{l}\text { Pré-competência } \\
\text { cultural }\end{array}$ \\
\hline & & $\begin{array}{l}\text { Negação/ } \\
\text { Indiferença cultural }\end{array}$ & $\begin{array}{l}\text { Reconheço as } \\
\text { limitações da minha }\end{array}$ \\
\hline & $\begin{array}{l}\text { Incapacidade } \\
\text { cultural }\end{array}$ & \multirow{3}{*}{$\begin{array}{l}\text { Não percebo a } \\
\text { importância da } \\
\text { diversidade cultural, } \\
\text { nem reconheço } \\
\text { as desigualdades } \\
\text { existentes. }\end{array}$} & $\begin{array}{l}\text { habilidade de } \\
\text { comunicação e tenho }\end{array}$ \\
\hline $\begin{array}{l}\text { Destrutividade } \\
\text { cultural }\end{array}$ & \multirow{3}{*}{$\begin{array}{l}\text { Estimo minha } \\
\text { cultura mais do que } \\
\text { as outras, não estou } \\
\text { disposto a ser útil } \\
\text { ou servir a outras } \\
\text { culturas, apresento } \\
\text { atitudes racistas, } \\
\text { mas não me esforço } \\
\text { conscientemente } \\
\text { para destruir a }\end{array}$} & & $\begin{array}{l}\text { consciência da minha } \\
\text { própria cultura e de } \\
\text { como ela interfere nas }\end{array}$ \\
\hline \multirow{2}{*}{$\begin{array}{l}\text { Me esforço } \\
\text { conscientemente } \\
\text { para destruir } \\
\text { culturas diferentes } \\
\text { da minha, porque } \\
\text { minha cultura é } \\
\text { tudo o que importa. }\end{array}$} & & & $\begin{array}{l}\text { minhas relações com } \\
\text { diferenças culturais. }\end{array}$ \\
\hline & & Ex.: Melting-pot theory & $\begin{array}{l}\text { Além disso, me } \\
\text { esforço para entender } \\
\text { como agir de maneira } \\
\text { culturalmente } \\
\text { competente. }\end{array}$ \\
\hline
\end{tabular}

Competência cultural

Tenho compromisso com a justiça social. Sou flexível e me esforço para me adaptar à cultura do outro, criando um ambiente útil e acolhedor a todos.

Proficiência cultural

Valorizo

significativamente a cultura do outro, sendo a base pela qual eu entendo as relações entre indivíduos, grupos, organizações, sistemas.

Sou capaz de interagir e me comunicar de maneira efetiva em grupos diversos.

Tuskegee. 
QuAdro 2

Possíveis erros no processo terapêutico em um contexto intercultural por Saldaña (2001 citado

por Martinez et al) La Competencia Cultural como referente de la Diversidad Humana en la

Prestación de Servicios y la Intervención Social, 2018. (traduzido pelos autores)

\section{Usuário}

Profissional

\section{Compromisso}

1. Percebe as diferenças culturais

2. Percebe o distanciamento social

3. Pensa que o profissional não deseja compreendê-lo

4. Teme ser julgado

5. Manifesta elevada ansiedade

1. Percebe as diferenças culturais

2. Percebe o distanciamento social

3. Segue entrevista clínica padronizada

4. Vê o usuário de maneira estereotipada

Aliança terapêutica
1. Não desenvolve um bom rapport
2. Se sente incompreendido
3. Demonstra muita desconfiança
4. Para de se revelar

\section{Resultado}

1. Demonstra frustração e ansiedade

2. Cancela as consultas de retorno

3. Falha nas explicações

4. Abandona prematuramente o tratamento
5. Manifesta aumento da ansiedade
1. Pensa que o usuário é resistente

2. Não compreende o usuário

3. Não responde adequadamente à desconfiança

4. Vê o usuário desmotivado profissionais de saúde para saírem de sua zona de conforto, no sentido de desenvolverem consciência do privilégio e desvantagem, racismo e preconceito presentes nas relações. E por este processo estar relacionado a um profundo entendimento da história dos grupos minorizados, do processo de colonização e de como isso impacta a saúde das populações que compõem o País hoje, e para além disso, utilizar esse entendimento de maneira positiva na construção das relações de cuidado ${ }^{28}$. Em geral, apesar de os profissionais de saúde possuírem boa capacidade de reflexão acerca de como seus valores influenciam as relações interpessoais (sensibilidade cultural), não apresentam atitudes culturalmente competentes e pouco conhecem sobre o cuidado de populações minorizadas sob sua responsabilida$\mathrm{de}^{35,36}$. Dessa maneira, além do treinamento para sensibilidade cultural, o acesso ao conhecimento teórico a respeito dessas populações e a um método de avaliação que considere aspectos atitudinais da competência cultural deveria ser incorporado ao currículo dos programas de residência em medicina de família e comunidade, visando à superação dessa dificuldade.

Para o desenvolvimento da competência cultural, a literatura sugere utilizar múltiplos cenários, contextos e estratégias pedagógicas que perpassem longitudinalmente e integrativamente o currículo, não devendo ser apenas uma disciplina, um estágio ou atividades isoladas, mas, sim, uma postura institucional. Existe a sugestão de incluir nos treinamentos consultas gravadas, simulações, discussões de casos, projetos com a comunidade e palestras/ treinamentos realizados por representantes de cada cultura minorizada ${ }^{29}$.
Embora a competência cultural seja reconhecida como uma importante estratégia para reduzir as disparidades culturais no cenário da saúde, faltam instrumentos validados e padronizados para avaliação. A maioria dos instrumentos são autorreferidos, norte-americanos e desenvolvidos pela enfermagem, conselhos e sistemas de saúde. Focam principalmente as habilidades culturais, autoconhecimento e conhecimento sobre outras culturas. Alguns ainda avaliam flexibilidade, abertura para receber a cultura do outro, resiliência emocional, desejo e sensibilidade cultural ${ }^{37}$.

\section{CONTRIBUIÇÕES PARA OS PROGRAMAS DE RESIDÊNCIA}

Tendo em vista as dimensões continentais do Brasil e sua diversidade, sugerimos que cada programa realize um diagnóstico cultural da população da sua região e então construa os objetivos de aprendizagem com base nas principais competências identificadas na revisão da literatura ${ }^{28,38}$, compiladas pelos autores segundo o domínio (Quadro 3).

Até o momento, não há uma sistematização de ensino da competência cultural em saúde no Brasil, seja na graduação ou pós-graduação, ocorrendo, na maioria das vezes, de maneira empírica e extracurricular ${ }^{34}$. Este ensaio compilou objetivos de aprendizagem encontrados na literatura, no intuito de colaborar com a sistematização do ensino desta competência por meio da inserção no currículo das residências de medicina de família e comunidade, respeitando-se as especificidades regionais. Ele também corrobora a necessidade de mudanças 
QuAdRO 3

Objetivos de aprendizagem para desenvolvimento da competência cultural, no contexto da atenção primária, segundo domínio

Domínio cognitivo

1. Demonstra conhecimento sobre a história, cultura,religião e situação de saúde de populações minorizadas sob seu cuidado

2. Demonstra conhecimento sobre o uso de plantas medicinais

3. Entende o impacto da colonização na desigualdade no acesso a serviços de saúde e nas barreiras culturais do Brasil

4. Entende as políticas de saúde do Programa Mais Saúde para ampliação do acesso a ações e serviços de saúde de qualidade por grupos minoritários

5. Identifica a si mesmo no continuum da competência cultural

6. Conhece os principais conceitos de cultura e suas principais características

7. Reconhece aspectos visíveis e invisíveis da cultura

\section{Domínio psicomotor}

1. Utiliza a abordagem centrada na pessoa como uma ferramenta para a competência cultural

2. Assegura que o usuário tenha explicações adequadas sobre o efeito e possível consequência do tratamento, incluindo alternativas às opções de tratamento propostas, com suas preferências sendo incluídas

Domínio atitudinal

1. Reconhece a espiritualidade como importante fator de saúde e bem-estar

2. Possibilita a expressão da espiritualidade pelo usuário para lidar com o sofrimento

3. Apresenta bom diálogo com outras figuras do cuidado da população, como pajés, curandeiras, rezadeiras, pastores e outros

4. Reconhece não ser o primeiro ou único cuidador a ser procurado pelo usuário

5. Demonstra consciência de seus próprios valores, crenças e suposições

6. Compreende que seus valores, crenças e suposições possuem impacto em sua prática

7. Fornece um ambiente adequado que melhor atenda às necessidades e interesses do usuário

8. Reflete sobre sua própria prática e valores que impactam a assistência em relação a idade, etnia, cultura, crenças, gênero, orientação sexual e/ou deficiência do usuário

9. Realiza cuidado compartilhado com membros de grupos culturais, conforme solicitado e aprovado pelo usuário

10. Ao exercer sua prática, respeita a identidade e o direito de cada pessoa de manter crenças, valores e objetivos pessoais

11. Evita agir de maneira preconceituosa e oferece advocacia quando o preconceito é aparente

12. Demonstra motivação para conhecer e compreender outras culturas

13. Reconhece o preconceito como prejudicial e limitante para as relações humanas

14. Realiza prática crítico-reflexiva, buscando enxergar novas e diferentes possibilidades

institucionais para que haja incentivo e valorização da prática da competência cultural, com disponibilização de tempo e infraestrutura para o desenvolvimento dessa competência pelos residentes de medicina de família e comunidade ${ }^{39}$. Além disso, foi identificada a necessidade de estudos futuros para a criação e/ou validação de instrumentos para avaliação direta da competência cultural no contexto brasileiro, uma vez que a maioria dos instrumentos são autorreferidos e sem tradução para o português ${ }^{40,41}$.

\section{REFERÊNCIAS}

1. Chancel L. World Inequality Report. 2018.

2. Gomes I, Marli M. Somos todos iguais? O que dizem as estatísticas. Retratos. 2018;14-9.

3. Watkins DA, Yamey G, Schäferhoff M, Adeyi O, Alleyne G, Alwan A, et al. Alma-Ata at 40 years: reflections from the Lancet Commission on Investing in Health. Lancet [Internet]. Elsevier Ltd; 2018;392(10156):1434-60. Recuperado de: https://linkinghub.elsevier.com/retrieve/pii/ S0140673618323894
4. Guimarães RCP, Cavadinha ET, Mendonça AVM, Sousa MF. Assistência a população LGBT em uma capital brasileira: o que dizem os Agentes Comunitários de Saúde? Tempus Actas de Saúde Coletiva [Internet]. 2017;11(1):121. Recuperado de: http:/ /www.tempus.unb.br/index.php/ tempus/article/view/2327

5. Ministério da Saúde. Política Nacional da população Negra. 3th ed. Brasília; 2017. 46 p.

6. Foronda C, Baptiste DL, Reinholdt MM, Ousman K. Cultural Humility: A Concept Analysis. J Transcult Nurs. 2014;27(3):210-7.

7. Heck JE, Sell RL, Gorin SS. Health care access among individuals involved in same-sex relationships. Am J Public Health. 2006;96(6):1111-8. /

8. Guimarães RCP, Cavadinha ET, Mendonça AVM, Sousa MF. Assistência a população LGBT em uma capital brasileira: o que dizem os Agentes Comunitários de Saúde? Tempus Actas de Saúde Coletiva [Internet]. 2017;11(1):121. Recuperado de: http://www.tempus.unb.br/index.php/ tempus/article/view/2327 
9. Ministério da Saúde. Saúde da População em Situação de Rua. 1st ed. Brasília;2014. 38p.

10. Ministério da Saúde. EDITAL DE CHAMADA PÚBLICA DSEI ALTO RIO JURUÁ. Brasília; 2018.

11. Starfield B. Atenção Primária: Equilíbrio entre necessidade de saúde, serviços e tecnologia. UNESCO, Ministério da Saúde, Brasília; 2002. 726 p.

12. Culturally competent general practice in New Zealand. In press 2006. Wellington: Royal New Zealand College of General Practitioners

13. Brasil. Mais Saúde: Direito de Todos 2008-2011. Brasília; 2010.

14. Brasil. Fundação Nacional de Saúde - Funasa. Política nacional de atenção à saúde dos povos indígenas. Brasília; 2002.

15. Pellon LHC, Vargas LA. Cultura, interculturalidade e processo saúde-doença: (Des)caminhos na atenção à saúde dos Guarani Mbyá de Aracruz, Espírito Santo. Physis. 2010;20(4):1377-97.

16. UNESCO. Biological aspects of race; a document of paramount importance. UNESCO Cour [Internet]. 1965; 4:p. 8-11.

17. Fletcher K. Are You Practicing Cultural Humility? - The Key to Success in Cultural Competence. California Health Advocates; Medicare: Policy, Advocacy and Education. 2007.

18. Spencer-Oatey, H. (2012) What is culture? A compilation of quotations. Global PAD Core Concepts. Available at Global PAD Open House http:/ / www.warwick.ac.uk/globalpadintercultura

19. Nursing Council of New Zealand. Competencies for entry to 308 Volume 33 Number 5, October 2006. Maori and Pacific Island Health the Register of Comprehensive Nurses. Wellington: Nursing Council Similarly

20. Weaver. Gary R. (1986). Understanding and coping with cross-cultural adjustment Stress. In R.M. Paige (Ed). Cross-cultural orientation. New conceptualizations and applications. Lanham MD: University Press of America.

21. Anand R, Lahiri I. Intercultural Competence in Health Care. In: Deardorff DK, organizador. The SAGE Handbook of Intercultural Competence. California; 2010. p. 387-402.

22. Campinha-Bacote J. (2002). The process of cultural competence in the delivery of healthcare services: A model of care. Journal of Transcultural Nursing, 13, 181-184.

23. Anand R, Lahiri I. Intercultural Competence in Health Care. In: Deardorff DK, organizador. The SAGE Handbook of Intercultural Competence. California; 2010. p. 387-402.

24. Tervalon M, Murray-García J. Cultural Humility Versus Cultural Competence: A Critical Distinction in Defining Physician Training Outcomes in Multicultural Education.
J Health Care Poor Underserved [Internet]. 1998;9(2):11725. Recuperado de: http://muse.jhu.edu/content/crossref/journals/journal_of_health_care_for_the_poor_and_ underserved/v009/9.2.tervalon.html

25. Foronda C, Baptiste DL, Reinholdt MM, Ousman K. Cultural Humility: A Concept Analysis. J Transcult Nurs. 2014;27(3):210-7.

26. Gurm BK, Cheema J. Cultural Safety Assessment of an Urban Canadian Hospital. Vol. 20, Journal of Cultural Diversity. 2013. p. 177-83.

27. Wepa D. Cultural safety in Aotearoa New Zealand. Second. Wepa D, organizador. Melborne: Cambridge University Press; 2005.

28. Watt K, Abbott P, Reath J. Developing cultural competence in general practitioners: an integrative review of the literature. BMC Fam Pract [Internet]. 2016;17(1):158. Recuperado de: http://bmcfampract.biomedcentral.com/ articles/10.1186/s12875-016-0560

29. Beach MC, Cooper LA, Robinson KA, Price EG, Gary TL, Jenckes MW, et al. Strategies for Improving Minority Healthcare Quality: Summary. 2004 Jan. In: AHRQ Evidence Report Summaries. Rockville (MD): Agency for Healthcare Research and Quality (US); 1998-2005. 90. Recuperado de: https: / /www.ncbi.nlm.nih.gov/books/NBK11918/

30. Lockyear PLB. Physician-patient communication: enhancing skills to improve patient satisfaction. Medscape Fam Med [Internet]. 2004;7. Recuperado de: http:/ /www.medscape.com/viewarticle/495199

31. Terrell RD, Lindsey RB. Culturally Proficient Leadership: The Personal Journey Begins Within. 2009. 1-174 p

32. Martínez MF, Martínez J, Calzado V. La Competencia Cultural como referente de la Diversidad Humana en la Prestación de Servicios y la Intervención Social. Psychosoc Interv [Internet]. 2006;15(3):331-50. Recuperado de: http:/ / scielo.isciii.es / scielo.php?script=sci_arttext\&pid =S1132-05592006000300007

33. Saha S, Beach MC, Cooper LA. Patient centeredness, cultural competence and healthcare quality. J Natl Med Assoc. 2008;100(11):1275-85.

34. Seeleman C, Suurmond J, Stronks K. Cultural competence: A conceptual framework for teaching and learning. Med Educ. 2009;43(3):229-37.

35. Seeleman C, Hermans J, Lamkaddem M, Suurmond J, Stronks K, Essink-Bot M-L. A students' survey of cultural competence as a basis for identifying gaps in the medical curriculum. BMC Med Educ [Internet]. 2014;14(1):216. Recuperado de: http://bmcmededuc.biomedcentral.com/ articles/10.1186/1472-6920-14-216 
36. Guerra O, Kurtz D. Building Collaboration: A Scoping Review of Cultural Competency and Safety Education and Training for Healthcare Students and Professionals in Canada. Teach Learn Med. 2017;29(2):129-42.

37. Shen Z. Cultural Competence Models and Cultural Competence Assessment Instruments in Nursing: A Literature Review. J Transcult Nurs. 2014 May 9. doi: $10.1177 / 1043659614524790$

38. Gupta SR. A Quick Guide to Cultural Competency: Practical Tools for Leading in Today's Multicultural Business Environment. Gupta Consulting Group. 2017. 44 p.

39. Taylor JS. Confronting "Culture" in Medicine's "CCulture of No Culture"”. Acad Med. 2003;78(6):555-9.

40. Gozu A, Beach MC, Price EG, Gary TL, Robinson K, Palacio A, et al. Self-administered instruments to measure cultural competence of health professionals: A systematic review. Teach Learn Med. 2007; 19 (2): 180-190. PMID: 17564547

41. Bernhard G, Knibbe RA, Von Wolff A, Dingoyan D, Schulz H, Mösko M. Development and Psychometric Evaluation of an Instrument to Assess Cross-Cultural Competence of Healthcare Professionals (CCCHP). PLoS One. 2015;10(12).

\section{CONTRIBUIÇÃO DOS AUTORES}

Eneline Gouveia liderou a elaboração do projeto, revisão bibliográfica, escrita do artigo. Rodrigo Oliveira contribuiu com a supervisão do trabalho e revisão do texto. Bruno Pessoa participou de todas as fases desde a elaboração do projeto, elaboração dos quadros e figuras, até a redação final.

\section{CONFLITO DE INTERESSES}

Os autores do presente estudo declaram não haver conflitos de interesse

\section{ENDEREÇO PARA CORRESPONDÊNCIA}

Eneline de Andrade Heráclio Gouveia

Secretaria de Saúde da Prefeitura do Recife

Residência de Medicina de Família e Comunidade

R. Doná Maria Augusta Nogueira, 519 - Bongi

Recife, BR 50751-53

email: eneline.gouveia@gmail.com 